\title{
Correction to: Holistic sustainability assessment of hybrid Al-GnP enriched nanofluids and textured-tool in machining of Ti-6Al-4V alloy
}

\author{
Aqib Mashood Khan ${ }^{1,2} \cdot$ Ghulam Hussain $^{3}$ - Mohammed Alkahtani ${ }^{4,5} \cdot$ Ayoub Alzabidi $^{4}$. Mustufa Haider Abidi ${ }^{5}$. \\ Ning $\mathrm{He}^{2}$
}

Published online: 4 December 2020

(C) Springer-Verlag London Ltd., part of Springer Nature 2020

\section{Correction to: Int J Adv Manuf Technol https://doi.org/10.1007/s00170-020-06371-x}

The original version of this article, unfortunately contained errors about funding and affiliation. The following is the list of errors and their correct presentation.

\begin{abstract}
Affiliation:
${ }^{1}$ Key Laboratory of High Efficiency and Clean Mechanical Manufacture, Ministry of Education, School of Mechanical Engineering, Shandong University, P.R. China.

${ }^{2}$ College of Mechanical and Electrical Engineering, Nanjing University of Aeronautics and Astronautics, Nanjing, 210016, China.

${ }^{3}$ Faculty of Mechanical Engineering, GIK Institute of Engineering Sciences \& Technology, Topi 23460, Pakistan

${ }^{4}$ Industrial Engineering Department, College of Engineering, King Saud University, Riyadh-11421, Saudi Arabia
\end{abstract}

The online version of the original article can be found at https://doi.org/ 10.1007/s00170-020-06371-x

Mohammed Alkahtani

moalkahtani@ksu.edu.sa

1 Key Laboratory of High Efficiency and Clean Mechanical Manufacture, Ministry of Education, School of Mechanical Engineering, Shandong University, Jinan, People's Republic of China

2 College of Mechanical and Electrical Engineering, Nanjing University of Aeronautics and Astronautics, Nanjing 210016, China

3 Faculty of Mechanical Engineering, GIK Institute of Engineering Sciences \& Technology, Topi 23460, Pakistan

4 Industrial Engineering Department, College of Engineering, King Saud University, Riyadh 11421, Saudi Arabia

5 Raytheon Chair for Systems Engineering (RCSE), Advanced Manufacturing Institute, King Saud University, Riyadh 11421, Saudi Arabia
${ }^{5}$ Raytheon Chair for Systems Engineering (RCSE), Advanced Manufacturing Institute, King Saud University, Riyadh11421, Saudi Arabia

Funding:

The authors are grateful to the Raytheon Chair for Systems Engineering (RCSE) for funding.

\section{Acknowledgment:}

The authors are grateful to the Raytheon Chair for Systems Engineering (RCSE) for funding. The authors are also thankful to Nanjing University of Aeronautics \& Astronautics, PR China, and the GIK Institute, Pakistan for technical assistance and resources provision. The authors would like to acknowledge the efforts made by Dr. Munish Kumar Gupta and Mr. Mozammel Mia for their valuable suggestion to improve the manuscript.

Publisher's note Springer Nature remains neutral with regard to jurisdictional claims in published maps and institutional affiliations. 